\title{
LA DISTINCIÓN HUMANO-ANIMAL EN LA MODERNIDAD FILOSÓFICA. UN ACERCAMIENTO A PARTIR DE LA CORRESPONDENCIA DESCARTES-MORE
}

\author{
Leticia Flores Farfán* \\ Rogelio Laguna*
}

En este artículo nos preguntamos cómo es que se ha conformado el alejamiento animal-humano en Occidente en el mundo moderno. Creemos que esta pregunta es fundamental si queremos valorar la pertinencia del debate contemporáneo acerca de los derechos animales, el Especismo y la ética ampliada. Partimos del debate respecto a la concepción de los animales en el debate Descartes-More, pues consideramos que gran parte de esa discusión sigue vigente nuestros días.

Palabras clave: Ética ampliada, siglo XVII, racionalidad, animales, antropocentrismo.

THE DISTINCTION HUMAN-ANIMAL IN THE PHILOSOPHICAL MODERNITY. AN APPROACH FROM THE CORRESPONDENCE DESCARTESMORE

\begin{abstract}
In this article we ask how the distance between the conception of human and animal was built in Occident in the XVII century. That is a fundamental question to evaluate the contemporary arguments and discussions about animal rights, Speciesism, and wide ethics. This article explains the discussion between Descartes-More in order to show how modern conception prevails in our days.
\end{abstract}

Keywords: Wide Ethics, XVII century, rationality, animals, anthropocentrism.

* Universidad Nacional Autónoma de México (UNAM), Ciudad de México, México. Correos electrónicos: leticiafloresfarfan@gmail.com,ralg23@yahoo.com.mx 


\section{Introducción}

Desde hace algunas déCAdAS SE han deSARRollado en la filosofía una serie de discusiones acerca del estatuto moral y jurídico de los animales, muchos de las cuales van de la mano de diversas investigaciones científicas que han arrojado importantes resultados sobre las capacidades emotivas e intelectuales de éstos ${ }^{1}$. Una de las discusiones más importantes es determinar qué tan cercanos o lejanos son en realidad los animales al ser humano, y en qué sentido podemos considerarlos, por ejemplo, poseedores de los mismos derechos.

La discusión sobre el acercamiento humano-animal ha sido un tema recurrente después del surgimiento de la teoría de la evolución y los estudios de genética comparada. Al menos desde la segunda mitad del siglo pasado, la filosofía ha tenido que preguntarse acerca de cómo incluir a los animales en el andamiaje teórico que ha construido acerca de la racionalidad como criterio de dignidad y de reconocimiento. El mayor problema es que ese andamiaje suponía, de inicio, una lejanía entre el ser humano y los animales que se había heredado, en gran sentido, de la Antigüedad, y se habría reformulado en la Modernidad, especialmente en el siglo XVII a partir de la filosofía mecanicista de René Descartes.

En este trabajo nos proponemos explicar por qué a partir de la concepción cartesiana los animales resultaron tan lejanos al hombre en el contexto filosófico, y cuáles son las concepciones más importantes que el mundo contemporáneo ha heredado de sus raíces modernas. Nos centraremos especialmente en la esclarecedora discusión sobre este tema presente en la correspondencia entre el vitalista neoplatónico de Cambridge, Henry More, y el filósofo francés paradigma de este nuevo periodo filosófico: René Descartes. Nos interesa rastrear y analizar los argumentos de ambos, para comprender cómo se estableció en el siglo XVII la distinción hombre-animal, que en muchos sentidos sigue siendo una referencia para el debate actual al respecto.

En un primer apartado estableceremos los aspectos más importantes de la doctrina cartesiana acerca de la filosofía natural, el segundo apartado lo dedicaremos específica-

1 Cfr. Regan, T., The Case for Animal Rights, University of California Press, Berkeley, 1983. 
mente al estudio de los animales en Descartes. A continuación, en el tercer apartado, presentaremos las críticas que More realiza a dicha concepción filosófica, específicamente en el caso de la concepción de los animales. Finalmente, junto a un recuento de los argumentos más importantes, estableceremos una posible respuesta a cuál es la herencia moderna en la concepción contemporánea sobre los animales.

\section{La filosofía natural cartesiana}

En la filosofía natural del filósofo de la Turena, particularmente en Los Principios de la Filosofía, el Discurso del Método y El Tratado del Hombre, se advierte que no hay distinción entre los seres artificiales (creados por el hombre) y los creados por Dios (seres naturales). Es decir, sin importar su origen, todos los seres se rigen por las mismas reglas naturales de composición y movimiento, por lo que, a nivel cuantitativo, no hay diferencia entre el cuerpo de un animal, del hombre o de una máquina. Así lo expresa también en el inacabado tratado Descripción del cuerpo humano, donde Descartes abiertamente establece una comparación entre el cuerpo humano y un reloj:

Trataré de dar cuenta de la máquina corporal entera, de tal forma que no haya razón alguna para creer que es nuestra alma la que produce, en ésta, los movimiento que, por experiencia, sabemos que no están controlados por nuestra voluntad, de la misma forma que no tenemos razón para pensar que hay un alma en el reloj que lo hace dar la hora ${ }^{2}$.

Descartes tiene claro que frente a los artificios humanos: relojes, fuentes, etc., los seres creados por la naturaleza resultan más complejos, e incluso tienen partes que no pueden percibirse por los sentidos ${ }^{3}$. Pero en términos explicativos Descartes advierte que los cuerpos, naturales o no, pueden comprenderse a partir de los principios de la máquina: causalidad, movimiento trasportado por contacto, engranaje de partes. La máquina como paradigma explicativo implica que no hay cuestión oculta alguna que explique la animación de las cosas. Todos los cuerpos tendrían una constitución común y ninguno de ellos es más importante ${ }^{4}$. En los Principios afirma:

Uno se persuadirá fácilmente que no hay alguna fuerza en las piedras o en las plantas de tal manera oculta, ningún milagro de la simpatía o la antipatía tan sorprendente, nada en fin en la naturaleza toda entera, que no pueda remitirse a causas corporales o al devenir del pensamiento y el espiritu, en las que la razón no pueda ser deducida de esos mismos principios $^{5}$.

2 Descartes, R., Descripción del Cuerpo Humano, AT XI, p. 226. [Para las obras de Descartes seguimos la citación canónica de acuerdo con la edición de Charles Adam y Pierre Tannery]

Descartes, R., Los Principios de la Filosofía, PRIN, VI, p. 203.

Rodis-Lewis, G., L'Antropologie Cártesienne, Presses Universitaires de France, Paris, 1990, p. 161.

Descartes R., Los Principios de la Filosofía, AT, VIII-1, p. 314, 29-315, 4. 
Aristóteles en su Política ya había comparado a los cuerpos con máquinas, pero había hecho una división entre máquinas animadas e inanimadas, que a Descartes no le resulta útil, puesto que la vida no es una propiedad geométrica de los cuerpos, y mucho menos una "fuerza oculta" que haya que desentrañar. Todas las funciones atribuidas a una posible "fuerza vital” bien pueden explicarse, según Descartes, recurriendo a leyes del movimiento, poleas, pesas, y agitación de los corpúsculos, el llamado mecanismo.

Debemos recordar -aunque no podemos desarrollarlo en este artículo- que el filósofo de la Turena explica la realidad a partir de un dualismo substancial (res cogitans-res extensa), expuesto en sus Meditaciones, a partir del cual distingue entre el cuerpo y el alma. El alma, dirá, además, es simple (contra la división tripartita del alma de Aristóteles), y su presencia implica la racionalidad del individuo, por lo que solo puede considerarse que se encuentra en el ser humano. Su simpleza implica que no pueda existir fragmentada en plantas y animales. Por lo tanto, el pensador francés considerará como un "error infantil" -como se verá en sus argumentos en la correspondencia con Henry More- que se le haya atribuido alma a dichos seres, mezclando sin cuidado lo corpóreo con lo espiritual ${ }^{6}$.

\section{Descartes y los animales}

Según lo que refiere Baillet ${ }^{7}$, el interés del filósofo sobre los animales habría surgido entre 1619 y 1625, cuando "todavía no tenía adversarios". Descartes habría esbozado un Tratado, clasificado tras su muerte en el inventario de Estocolmo como Thaumantis regia, en el que se interesaba por la mecánica antigua, los artificios maravillosos y los efectos ópticos. En ese entonces, Descartes habría formulado su ya conocida postura sobre los animales-autómatas, la que iría completando y reformulando en años posteriores.

Mientras escribía el Mundo, Descartes le expresa a Mersenne desde Deventer (junio de 1632) su interés inicial por hablar sobre la generación de los animales, preocupación que finalmente abandona:

Durante un mes he estado indeciso sobre si describir cómo se produce la generación de los animales en mi Mundo; al fin he decidido no hacer nada, porque la cosa requería demasiado tiempo. He acabado pronto todo lo que había programado introducir en torno a los cuerpos inanimados; no me queda sino añadir alguna cosa sobre la naturaleza del hombre. Después la transcribiré para enviárselo... ${ }^{8}$.

6 Sobre este tema hay muchas referencias: se puede consultar, por ejemplo, la carta del 22 de junio de 1641 . Descartes, R., Meditaciones Metafísicas, AT, VII, p. 26, 6-8. También Correspondencia Descartes-More, p. 75. (Descartes a More, 5 de febrero de 1649) Un antecedente sobre esa tesis, que es probable que Descartes no conociera, es un texto de Gómez Pereira, médico español de la segunda mitad del siglo XVI, que ya había afirmado que no se pueden tomar los signos exteriores como pruebas de sensibilidad e inteligencia. Cfr. BAILLET, Vie de M. Descartes, I, pp. 51-52, referido en Garin, E., Descartes, trad. de José Martínez G., Crítica-Grijalbo, Barcelona, 1989, p. 62.

7 Baillet, Vie..., op. cit., I, pp. 51-52, referido en Garin, E., op. cit., p. 62.

8 GARIN, E., op. cit., p. 94. 
Posteriormente, Descartes introducirá algunas referencias sobre los animales, donde ya los considera meramente corpóreos y sujetos a las leyes de la física mecanicista. Principalmente abordará el tema, aunque de forma breve, en el Discurso y en el Tratado del Hombre.

En el Discurso, por ejemplo, escribe:

Esto no extrañará a quienes saben que la habilidad del hombre le permite construir con muy pocas piezas una diversidad de autómatas o máquinas que se mueven, en comparación, con la gran cantidad de huesos, músculos, nervios, arterias, venas y todas las otras partes que están en el cuerpo de un animal. Ellos considerarán este cuerpo como una máquina que, siendo construida por las manos de Dios, está incomparablemente mejor ordenada que cualquier máquina hecha por el hombre y que contiene movimientos mucho más maravillosos que los de cualquier máquina9.

El 20 de febrero de 1639 Descartes expresa su interés por los animales para comprender cómo está constituida la corporalidad del hombre. En una carta de la fecha mencionada el pensador le dice a Mersenne que la máquina del cuerpo le ha interesado desde siempre y que desde once años atrás no había hecho sino diseccionar los más diversos animales ${ }^{10}$, incluso yendo más allá que Vesalio y otros anatomistas.

El 13 de noviembre del mismo año, escribe:

Debe tener un espiritu bien estrecho aquella persona de quien me escribe, que me acusa de ir por los pueblos para ver matar los cerdos [...] No es un delito tener curiosidad por la anatomía; durante un invierno en Ámsterdam iba todos los días a casa de un carnicero, para verlo cuando mataba a los animales; y hacía llevar desde alli a mi casa las partes que quería diseccionar más a mi comodidad; cosa que he hecho frecuentemente en los lugares donde he estado ${ }^{11}$.

En sus diversas reflexiones observamos que el pensador considera que los animales se pueden explicar bajo los principios de las máquinas -como el resto de los cuerpos del universo físico-. Los animales son autómatas sin conciencia -aunque Descartes les reconocerá sensación, como explicaremos más adelante-. Esto quiere decir que los animales,

9 Descartes, R., AT VI, p. 56.

10 Sobre las disecciones recuérdese aquella famosa anécdota referida por los biógrafos (Baillet, Vie..., op. cit., T. II, p. 273) y por el propio Descartes (Carta del 20 de febrero de 1658, AT, V, p. 353), que habría pasado en Egmond en 1653 en el que uno de sus amigos, visitando a Descartes, le habría solicitado conocer sus libros en los que había hecho sus estudios física y a los que recurría más frecuentemente. Descartes guió al hombre por un largo pasillo y le mostró un becerro, a cuya disección se ocuparía al día siguiente.

11 GARIN, E., op. cit., p. 121.

12 Leibniz recuperara esta noción en su Cartesius, donde observará que hay "leyes naturales" en los cuerpos, que explican, por ejemplo, por qué ciertos animales lanzados al agua se equilibran naturalmente, mientras que el hombre tiene que agitarse y nadar. $C f r$. Rodis-Lewis, G., op. cit., p. 164. 
como todos los cuerpos, son susceptibles de ser descritos como mecanismos sujetos a las reglas naturales universales ${ }^{12}$. "Decir que el animal es una máquina, es equivalente a afirmar que su operación se rige por las reglas naturales comunes a la operación de toda entidad material", explica Alejandra Velázquez ${ }^{13}$. Si bien, admite Descartes, los animales nos sorprenden a veces en su diseño, no dejan de ser mecanismos.

Cabe destacar que, al momento de considerar la anatomía de los animales y también del hombre, Descartes sigue el programa que había establecido en la Regla XII, en la que había solicitado como parte del método de conocimiento que se dividiera el objeto de conocimiento en las partes más pequeñas y simples que fuera posible. El estudio del cuerpo, a través de las partes que la conforman, es una continuación de dicha solicitud ${ }^{14}$. Esta misma intención continuará en las Pasiones, aplicada al caso del hombre ${ }^{15}$.

En su carta a Henry More del 5 de febrero de 1649 -que retomaremos en la siguiente sección- Descartes sostiene: “...parece razonable que la naturaleza produzca sus propios autómatas, los cuáles son mucho más espléndidos que los artificiales"16. Estos autómatas, los animales, son máquinas en movimiento y en tanto tales no tienen pensamientos. Así, la ausencia de pensamiento marca la distancia de los animales con el ser humano.

En el Discurso del Método, Descartes ya había expresado que si bien el hombre comparte con los animales -y con los cuerpos en general- el poseer un cuerpo mecánico, difiere de cualquier mecanismo, y de los animales, principalmente, por ser capaz de valerse del lenguaje $\mathrm{e}^{17}$, mientras que los animales y las máquinas son indistinguibles ${ }^{18}$. Nuestro autor escribe: "[si unas máquinas] tuvieran órganos y el aspecto exterior de un mono o de cualquier otro animal que carece de razón, no tendríamos manera alguna de saber que éstas no poseen la misma naturaleza que estos animales"19.

Ante la confusión que genera que los cuerpos animales y humanos presenten semejanzas, Descartes explica en las Pasiones que tanto hombres como animales comparten los movimientos de los espíritus animales ${ }^{20} \mathrm{y}$ de la glándula pineal, pero en los animales dicha semejanza no tiene consecuencias en el ámbito de las pasiones, sino en los movimientos de los músculos y los nervios ${ }^{21}$.

13 Velázquez, A., "Las ideas cartesianas acerca de la sensación en los animales", en: Cuaderno de Historia de la Filosofía, Proyecto Filosofía Moral y Filosofía Natural, IIF-UNAM, México, UBA, Buenos Aires, Primavera 2001, No. 4, p. 17.

14 Aucante, V., La Philosophie Médical de Descartes, Presses Universitaires de France, Paris, 2006, p. 49.

15 Descartes, R., Las Pasiones del Alma, art. 7, AT, XI, p. 331; art. 9, p. 332, 31.

16 Idem., AT V, p. 366.

17 Como después retomó Malebranche, el leguaje nos indica que los demás hombres no son meros autómatas. "La libertad se manifiesta por la negación como por el uso positivo de los signos". Cfr. Rodis-Lewis, G. op. cit., p. 37.

18 Cfr. Monroy, Z., "Pensamiento animal”, en: Benítez, L.- Robles, J.A. (Comps.), Homenaje a Margaret Wilson, Universidad Nacional Autónoma de México, Instituto de Investigaciones Filosóficas, México, 2002, p. 89.

19 Descartes, R., AT VI, p. 56.

20 Partículas corpóreas que transmiten ciertos movimientos del cerebro al cuerpo.

21 Descartes, R., Las Pasiones del Alma, XI, pp. 369-370. 
En su correspondencia con William Cavendish ${ }^{22}$, Marqués de Newcastle, Descartes deja claro que a pesar de la semejanza de los órganos corporales, esto no implica que tengan pensamientos ${ }^{23}$ como nosotros $^{24}$ :

Si se le enseña a una cotorra a decir buenos días a su dueña, al verla aproximarse, esto sólo puede hacerse convirtiendo la emisión de la palabra en la expresión de una de sus pasiones. Por ejemplo, será la expresión de su esperanza de comer, si siempre se le ha dado un bocado cuando dice la palabra. De forma similar, todos los actos que se enseñan a los perros, caballo y monos son sólo la expresión de su miedo, su esperanza o su alegría [...] Pero el uso de palabras, como tales, es propio de los seres humanos ${ }^{25}$.

Si se admitiera que éstos tienen pensamientos, de eso mismo se seguiría que tendrían un alma inmortal como nosotros, lo cual es inverosímil pues "no habría razón de creerlo en algunos animales sin creerlo de todos y muchos de ellos, como las ostras y esponjas, etc., son demasiado imperfectos para que esto sea creíble" ${ }^{26}$. Además, como ya mencionamos, la cuestión del lenguaje muestra la capacidad interpretativa del hombre, mientras que los animales se dejan llevar por las primeras impresiones, lo que prueba la libertad del hombre y la mecánica de los animales ${ }^{27}$. Es cierto que hay cosas que los animales hacen mejor que el hombre -explica Descartes al marqués-, la diferencia es que éstos lo hacen respondiendo a sus mecanismos, como un reloj que da la hora con precisión y no por la acción del alma ${ }^{28}$.

Ahora bien, respecto a la sensación, Descartes no mantiene una misma postura ${ }^{29}$, la cual sufre un viraje conforme madura su obra. En una primera etapa, nuestro autor considera que, a causa de la ausencia del alma, los animales no pueden experimentar sensaciones, por ejemplo, dolor. Esto lo refiere Descartes en una carta a Mersenne hacia 1640:

No explico sin alma la sensación de dolor, pues para mí el dolor no está más que en el entendimiento; empero, explico (fisiológicamente) todos los movimientos exteriores que nos acompañan en esa sensación, los cuales se encuentran en las bestias, y no el dolor propiamente dicho ${ }^{30}$.

Bajo esta explicación, los animales al carecer de alma, carecen de sensación ${ }^{31}$. El filósofo explica en enero de 1642 a Gibieuf, que los movimientos de los animales, a pesar de

22 Cavendish (1592-1670) fue un defensor de la monarquía que se refugió en Holanda, donde compartió intereses científicos y filosóficos con Descartes. Descartes v/s Montaigne los animales no piensan.

23 Rodis-Lewis piensa que, si bien Descartes en su correspondencia cuida la sutileza de sus argumentos al negar el alma de los animales, sus discípulos y seguidores van a afirmar dicha cuestión con todas sus letras.

24 Cfr. Descartes, R., Discurso del Método, AT VI, p. 56.

25 Idem., AT IV, p. 574.

26 Idem., IV, p. 576. Cfr. Monroy, Z., "Pensamiento animal", op. cit., p. 89.

27 Descartes, R., Las Pasiones del Alma, art. 138. Cfr. Rodis-Lewis, G., op. cit., p. 37 y p. 75.

28 Descartes, R., AT, IV, p. 575. Cfr. Monroy, Z., "Pensamiento animal", op. cit., p. 89.

29 VelázQuez, A., op. cit., p. 17.

30 Descartes, R., AT III, p. 85. 
ser semejantes a los humanos, se pueden realizar sin sensación y sin imaginación:

Por lo que toca a los animales, notamos que aunque en ellos los movimientos [son] semejantes a aquellos que se siguen de nuestra imaginación o sensaciones, no por ello [se siguen] de la imaginación o sensaciones. Y al contrario, esos mismos movimientos se podrian también hacer sin imaginación... ${ }^{32}$.

Este punto de la propuesta cartesiana fue, sin duda, un tema controversial que continúa abierto hasta la fecha. Críticos contemporáneos como Peter Singer ${ }^{33}$ llegan incluso a buscar en Descartes al responsable por el maltrato animal.

Sin embargo, la opinión de Descartes no es definitiva, y en febrero de 1649 establece una nueva perspectiva para este asunto:

[...] Aunque considero que está establecida nuestra incapacidad para probar la existencia del pensamiento en los animales, [...] Nótese por favor que estoy hablando de pensamiento y no de vida ${ }^{34}$ o sensación. No niego la vida a los animales, desde que considero que ésta consiste en el calor del corazón, y no niego aun la sensación, tanto como ésta depende de un órgano corpóreo ${ }^{35}$.

Descartes admite la sensación y la vida en los animales, pero, como veremos a continuación, este hecho no implica un cambio en el rumbo de lo que ha dicho sobre los animales; más bien parece afinar la doctrina y resolver algunos problemas explicativos sobre la fisiología animal. Esto parece quedar indicado en la carta que Descartes dirige a Henry More en febrero de 1649, donde señala dos fuentes del movimiento corpóreo:

El primero es puramente mecánico y corpóreo y depende solamente de la fuerza de los espíritus y de la estructura de nuestros órganos y puede denominarse alma corporeizada. El otro, un principio incorpóreo, es la mente o el alma, que he definido como sustancia pensante $^{36}$.

Así, Descartes establecerá una clasificación de distintos tipos de percepción -sobre todo en vías de dar cuenta del movimiento del hombre-, que pueden explicar por qué los

31 Malebranche dirá, desde su interpretación del cartesianismo, que los gritos de los animales son resultado de sus mecanismos. Un hombre, en cambio, puede sentir dolor y resistirse a gritar, puede resistirse al juego de su máquina. Rodis-Lewis, G., op. cit., p. 77.

32 Descartes, R., AT III, p. 47.

33 Singer, P., "Los animales y el valor de la vida", trad. de Alejandro Herrera, en: Kwiatkwoska, T.- Issa, J. (Comps.), Los Caminos de la Ética Ambiental, Universidad Autónoma Metropolitana, Plaza y Valdés, 1998, p. 205. Hay que reconocer, sin embargo, que el propio Singer matiza su crítica señalando que la interpretación que permite la crueldad hacia los animales bajo el argumento, por ejemplo, de que no sienten dolor tal vez no es lo que Descartes quería señalar al respecto.

34 Descartes, R., AT V, pp. 276-278.

35 Idem., AT V, p. 278.

36 Cfr. Velázquez, A., op. cit., p. 18. 
cuerpos animales corresponden, efectivamente, a un mero registro fisiológico de estímu$\operatorname{los}^{37}$. La afirmación de que los animales tienen sensación no sería, entonces, una nueva concepción de la "vida" animal.

En las Respuestas ${ }^{38}$ a las Sextas Objeciones Descartes distingue los tres niveles de percepción:

1) La perspectiva física o fisiológica de la sensación

2) La conciencia de la sensación (enlace físico-mental)

3) La sensación de operaciones complejas mentales en la base de la interacción mentecuerpo.

Para Laura Benítez, Descartes intenta separar los aspectos mecánicos de los intelectuales para distinguir: la sensación, la experiencia de la sensación y el juicio acerca de la sensación, especialmente porque lo que le interesa es comprender y explicar el conocimiento humano.

En el caso de los animales, éstos se quedarían únicamente al nivel de la mera sensación (nivel 1), como explica la carta a More antes citada, nivel que puede explicarse mecánicamente como un efecto de los objetos sobre los órganos del cuerpo, por el choque de las partículas. Esto, -explica Benítez-, es lo que comparten animales y seres humanos. Los otros dos niveles, que corresponden a tener o experimentar sensaciones, son algo estrictamente humano, en tanto que la conciencia es una operación anímica, facultad de la que están desprovistos los animales. El tercer nivel, el establecer juicios al respecto de las sensaciones y poder corregir los errores de la percepción sensible, será una posibilidad propiamente humana.

Si bien la división anteriormente establecida por Descartes permite entender con mayor claridad la compleja relación existente entre el cuerpo humano y su principio anímico, nos deja - como acertadamente lo señala Alejandra Velázquez- ciertas interrogantes: “qué implica aceptar que los animales poseen sensación?, ¿sienten acaso, dolor, en el sentido en que entendemos esta expresión los seres humanos? [...] ¿en qué se distingue el tener sensaciones de tener conciencia de las mismas" ${ }^{39}$.

37 "Para comprender mejor cuál es la certeza de los sentidos, es necesario distinguir en ellos tres clases de grados. En el primero, no debemos considerar otra cosa sino lo que los objetos externos causan inmediatamente en los órganos corpóreos [...] El segundo contienen todo lo que resulta inmediatamente en el espíritu, por estar unido a los órganos corpóreos [...] tales son las sensación es de dolor, cosquilleo, hambre, sed, colores, sonidos, sabores, olores, calor, frío y otros semejantes que [..., provienen] de la mezcla del espíritu con el cuerpo. Y finalmente, el tercero comprende todos los juicios que tenemos costumbre de hacer [...] con ocasión de las impresiones o movimientos que realizan en los órganos de nuestros sentidos. Descartes, R., AT VIII, p. 237/AT IX-1, p. 236, trad. de Laura Benítez.

38 VelázQuez, A., op. cit., p. 19.

39 Idem., p. 20. 
Estas preguntas reflejan el serio problema que resulta ser el cuerpo vivo en la filosofía cartesiana, especialmente al comparar la realidad de otros seres vivos en relación con la experiencia humana de la corporalidad. Para Descartes, al parecer, sólo podemos aspirar a describir las operaciones fisiológicas con las que operan los organismos ${ }^{40}$. Más allá de esto, poco más puede explicarse. Velázquez apunta que esta dificultad resulta del hecho que Descartes evita comparar la realidad humana con la de otros seres vivos ${ }^{41}$, es decir, parece que frente a los animales estamos en un ámbito inconmensurable que Descartes no antropomorfiza ${ }^{42}$.

\section{La discusión Descartes-More}

La propuesta cartesiana sobre los animales fue difundida rápidamente en su época, y fue recogida, desde intereses propios, por los materialistas franceses y por algunos médicos, generándose, en ese movimiento, un nuevo paradigma de la animalidad mecánica -establecida también en el cuerpo humano-. Aunque debe tenerse claro que las voces críticas tampoco faltaron, especialmente de una de las corrientes que se desarrollaba paralelamente al mecanicismo cartesiano y que tendría resonancias hasta el darwinismo: el neoplatonismo de Cambridge.

Los neoplatonistas de Cambridge, que expresaban una filosofía de carácter vitalista, tenían una serie de argumentos propios con los que enfrentaron aspectos particulares de la filosofía cartesiana, como fue el caso de la concepción de los animales. Un registro de esta discusión entre doctrinas explicativas quedó plasmada en la discusión epistolar que Descartes sostuvo con Henry More entre 1648 y 1649. More, nacido en 1614, era más joven que Descartes. El neoplatonismo y su rechazo al calvinismo, practicado fervientemente por su familia, eran los dos polos fundamentales en los que había establecido su doctrina. Simpatizaba, hasta cierto punto, con el cartesianismo en su defensa permanente del dualismo entre el espíritu y la materia ${ }^{43}$, aunque, a diferencia de Descartes, aceptará mediaciones entre ambos elementos del dualismo, lo que le implicaría reconocer diversos grados de racionalidad para establecer una inteligencia animal.

En 1646, mientras More está interesado en realizar una filosofía natural estrechamente vinculada con la teología, se acerca a la filosofía cartesiana leyendo con gran interés Los Principios de la Filosofía. More encuentra en Descartes un respaldo teórico a la tesis de

40 Ibidem.

41 Es importante dejar anotada esa vía para pensar un Descartes que no es antropocéntrico a ultranza, y no sólo respecto a los animales.

42 GonZÁlez Recio, J.L., "La seducción por el espacio y la morada del espíritu”, en Correspondencia DescartesMore, p. 11.

43 J.L. González Recio explica que en la propuesta de More no encontramos tampoco una restauración de la teoría aristotélica de los seres vivos, "sino [que estamos] ante un platonismo tamizado por la psicología racional. Psicología racional y teodicea que siempre están ligadas a la emoción y a la sensibilidad religiosa. Idem., p. 16. 
que las explicaciones naturales son subsidiarias de las explicaciones metafísicas. Si bien posteriormente More se alejará de Descartes, será uno de los principales responsables de la difusión de la filosofía cartesiana en Gran Bretaña, enseñando, por ejemplo, dicha filosofía en Cambridge.

La correspondencia surge a partir de este primer acercamiento en el que More, con las debidas reservas, considera que su sistema y el cartesiano comparten elementos fundamentales, a saber, el dualismo, y la relación entre las explicaciones naturales y las metafísicas. Sin embargo, como se muestra en la correspondencia a la que a continuación referiremos, el alejamiento entre ambos filósofos se lleva a cabo cuando se advierte la diferencia en cómo comprendía cada uno el dualismo y la relación entre materia y espíritu. De esto deriva el fuerte desencuentro teórico respecto a la comprensión de la vida, especialmente los animales, pues para Descartes ésta era explicada a partir del corpuscularismo y las leyes mecánicas de movimiento, mientras que More acepta la existencia de un espíritu de la naturaleza que, sin ser material, es extenso y constituye el origen de la vitalidad de todos los seres ${ }^{44}$, por ejemplo los animales; tesis, que resultaba ridícula para Descartes, no sólo por oscura, sino porque era contraria a su dualismo radical.

La correspondencia entre ambos pensadores consta de cuatro cartas de More y tres de Descartes, aunque debe tenerse en cuenta que Descartes sólo envío dos y preparó el borrador de la tercera: su muerte le impidió concluir la tercera misiva, que Clerselier le hizo llegar a More en 1655.

Dicha correspondencia es amplia en las preocupaciones y vías argumentativas. Para los propósitos de este trabajo nos detendremos en un comentario de More en la primera carta que dirige al francés el 11 de diciembre de 1648. Después de discutir acerca de la impenetrabilidad y extensión de la materia, le advierte que más allá de lo dicho con anterioridad, la objeción de la que más "se rebela mi espíritu" es contra "el sentimiento homicida y bárbaro que anticipáis en vuestro Método y por el que despojáis de vida y de sentimiento a todos los animales; o más bien sostenéis que nunca han gozado de ellos; pues no sabréis aceptar que hayan vivido nunca" $"$.

Más adelante More sigue desarrollando su argumento en un tono de fuerte reproche:

En este punto las luces penetrantes de vuestro espíritu me causan menos admiración que espanto: alarmado por el destino de los animales considero menos en vos esta sutilidad ingeniosa, que ese hierro cruel y cortante del que parecéis armado para arrancar de un solo golpe la vida y el sentimiento de todo lo que es casi animado en la naturaleza, para transformarlo en estatuas de mármol y en máquinas ${ }^{46}$.

44 Correspondencia Descartes-More, p. 49. (More a Descartes, 11 de diciembre de 1648).

46 Monroy, Z., "Pensamiento animal”, op. cit., p. 89. 
En este punto Zuraya Monroy explica -siguiendo a Margaret Wilson- que las dificultades que More tiene con la propuesta mecanicista de Descartes y las consecuencias de ésta en la vida animal, resultan justamente de que More parece alarmarse de que con el mecanicismo el francés estaría privando a los animales de toda vida. Pero ¿por qué More lo entendió así?, se pregunta la comentadora y reflexiona: "me parece que él expresa la antigua convicción de que el alma es lo único que puede dar vida" ${ }^{37}$.

Más adelante en la carta, y en un tono más argumentativo, More le pregunta a Descartes si "¿sería posible que los loros o las cotorras pudieran imitar nuestros sonidos, si no entendieran y no percibieran por sus órganos lo que decimos?" ${ }^{48}$, ya que, para More, el habla de estos animales va más allá de la imitación, de hecho, cuando tienen hambre son capaces de pedir comida ${ }^{49}$.

Si es cierto que, como dice Descartes, los animales son meros mecanismos y carecen de un principio vital -que, por el contrario, More encuentra desplegado por doquier en la Naturaleza-, de una vida interior, y de razón, se pregunta el inglés:

¿De dónde podría proceder sin ello la sutileza y sagacidad de los zorros y los perros? ¿A qué se debe que las amenazas las palabras sean capaces de contener a las bestias cuando muestran su ferocidad? ¿Por qué cuando un perro hambriento ha robado algo y se esconde como si supiera que ha obrad mal, y caminando con temor y desconfianza, no saluda a nadie al pasar, sino que, acortando su camino, busca con la cabeza baja un lugar apartado valiéndose de una sabia precaución, para no ser castigado por su crimen? ¿Cómo explicar todo esto sin un sentimiento interior? La lista infinita de relatos que podemos elaborar para demostrar que los animales poseen razón ¿no sirve al menos para probar que poseen sentimiento y memoria?[...] No me cabe duda de que existen gran cantidad de hechos que denotan por sí mismos una fuerza y una sutileza de espíritu, que está más allá de la materia y que no podríamos eludir ${ }^{50}$.

Muchas de las preguntas de More son falsos problemas para el sistema cartesiano, construido desde un inicio en la diferencia sustancial y donde no es necesaria la existencia de un principio vital para explicar el movimiento de los cuerpos, salvo en el caso del ser humano. No pasa inadvertido para los comentadores contemporáneos que More hace las preguntas correctas y que apunta a cuestiones problemáticas de la doctrina cartesiana, especialmente en su opinión respecto a los animales.

More expone que cree saber cuál es el origen del rechazo de Descartes a reconocer un principio vital y la razón en los animales: "el motivo [...] es la inmortalidad del alma que

47 Correspondencia Descartes-More, p. 49. (More a Descartes, 11 de diciembre de 1648).

48 Idem.

49 Correspondencia Descartes-More, p. 51. (More a Descartes, 11 de diciembre de 1648).

50 Idem., p. 53. (More a Descartes, 11 de diciembre de 1648). 
habéis querido establecer". [Además] Al haber supuesto que el cuerpo era incapaz de pensar, habéis concluido que allí donde hubiera pensamiento debía existir una sustancia realmente distinta del cuerpo, y en consecuencia inmortal; de donde se sigue que si las bestias pensaran, tendrían almas, que serían sustancias inmortales" 51 . A pesar de lo anterior, no le queda claro a More por qué Descartes prefiere hacer de ellos "máquinas inanimadas, antes que cuerpos movidos por almas inmortales" ${ }^{2}$.

Descartes responde el 5 de febrero de 1649, desde Egmond, a los argumentos de More, y le indica, después de diversas cortesías epistolares, que creer que los animales piensan es el mayor de todos los prejuicios que "hemos retenido de nuestra infancia" 53 . Esto es así, explica, por pensar que los miembros de los animales eran semejantes a los nuestros, al igual que sus movimientos, y de ello haber concluido falsamente que debían poseer un alma como nosotros que dotaba a su cuerpo de movimiento ${ }^{54}$. Este prejuicio se supera, sin embargo, cuando, tras haberlo meditado mucho tiempo, nos percatamos que existen dos fuentes que originan nuestro movimiento: "uno completamente mecánico y corporal, que sólo depende de la fuerza de los espíritus animales y de la configuración de las partes, y al que podríamos llamar alma corporal, y el otro incorpóreo, es decir, el espíritu o el alma"55.

Descartes advierte a More que ha investigado cuidadosamente de cuál de aquellos dos principios proviene el movimiento de los animales, y que ha comprobado que proceden de uno solo, del corporal y mecánico, puesto que "he tenido por demostrado que no podíamos probar de ningún modo que hubiera en los animales un alma que pensara" ${ }^{56}$. Sin tener que explicar la astucia de los zorros o perros, ni detenerse en lo que hacen los animales para conseguir comida, dice nuestro autor, basta observar los miembros de los animales para llegar a esa conclusión, toda vez que es más probable hacer "que se muevan como máquinas los gusanos de la tierra, las moscas, las orugas, y los demás animales, que otorgarles un alma inmortal"57. Luego, como Descartes no acepta (como More) que exista alguna mediación entre la sustancia pensante y la extensa, no puede existir tampoco una suerte de gradación en los animales: si el alma racional no puede ser aplicada a un gusano, no habría razón para aplicarla a un mamífero.

Recordemos que Descartes ya había expresado en su Tratado del Hombre y en el Discurso que los movimientos y funciones del cuerpo podían explicarse sin recurrir al alma, puesto que todas estas funciones obedecían a mecanismos y a la disposición de palancas, ruedas y contrapesos. En esta máquina:

51 Ibidem.

52 Correspondencia Descartes-More, p. 75. (Descartes a More, 5 de febrero de 1649).

53 Idem.

54 Ibidem.

55 Correspondencia Descartes-More, p. 77. (Descartes a More, 5 de febrero de 1649).

56 Idem.

57 Descartes, R., Tratado del Hombre, AT XI, p. 202. 
[...]no es necesario concebir en ella ninguna otra alma vegetativa o sensitiva o cualquier otro principio de movimiento y vida, aparte de su sangre y sus espiritus, agitados por el calor del fuego que arde continuamente en su corazón, fuego que tienen la misma naturaleza de todos los fuegos que aparecen en los cuerpos inanimados ${ }^{58}$.

A nivel anatómico, sigue Descartes en su carta, tanto en el cuerpo de los animales como en el nuestro hay nervios, músculos, sangre, espíritus animales y otro órganos "dispuestos de tal modo que pueden producir por sí mismos, sin el auxilio de ningún pensamiento, todos los movimientos que observamos en los animales" 59 . Así como el hombre -prosigue el filósofo de la Turena- es capaz de construir autómatas que no requieren de la razón para moverse, así la naturaleza "puede por su parte producir esos autómatas y muchos más excelentes como los animales" ${ }^{60}$. Lo anterior nos persuade, según Descartes, de que tengamos que suponer el pensamiento en todas partes donde veamos una conformación de miembros semejantes a la de los animales. Aunque la razón principal es que, aunque algunos animales tienen disposición para retener lo que se les enseña y tienen movimientos más o menos perfectos, ningún animal ha llegado a "hacer uso de un auténtico lenguaje [...] pues la palabra es el único signo y la única marca segura de la existencia del pensamiento oculto y encerrado en el cuerpo"61.

Descartes, a continuación, hace una distinción única en el resto de su obra: “...hay que señalar, sin embargo, que estoy hablando del pensamiento, no de la vida, o el sentimiento; pues yo no le niego la vida a ningún animal, al hacerla consistir sólo en el calor del corazón. Tampoco les niego el sentimiento en tanto que este depende de los órganos del cuerpo" ${ }^{\circ}$. ¿Cuál es esa vida que admite Descartes? Según lo que indica el pasaje se trata únicamente de reconocer un proceso fisiológico, que no implicaría de fondo salir del mecanicismo, aunque hacer este reconocimiento, seguramente gatillado por los reproches de More, podría implicar que Descartes advierte la necesidad de realizar una distinción entre ciertos cuerpos y la materia propiamente dicha. Dicha distinción sin embargo no se llevó nunca a cabo. No obstante, contra More, el filósofo de la Turena deja clara la preocupación central de su trabajo: dejar fuera de la explicación del cuerpo las doctrinas que atribuían a elementos del alma o principio vital ciertas funciones, ya que esto resulta oscuro para el entendimiento y fuera del alcance explicativo de la física ${ }^{63}$.

El 5 de marzo de 1649 More responde a la misiva de Descartes, convirtiéndose la discusión en un diálogo de sordos respecto al problema en el que nos hemos enfocado y aquel "prejuicio de la infancia": "yo experimento en mi la fuerza de ese prejuicio más

58 Correspondencia Descartes-More, p. 79. (Descartes a More, 5 de febrero de 1649).

59 Idem.

60 Correspondencia Descartes-More, p. 81. (Descartes a More, 5 de febrero de 1649).

61 Idem.

62 Monroy, Z., "Pensamiento animal”, op. cit., p. 90.

63 Correspondencia Descartes-More, p. 107. (More a Descartes, 5 de marzo de 1649). 
allá de todo lo que os pueda decir, y me siento tan preso y tan paralizado en sus redes que me resulta imposible desembarazarme de él". En términos argumentativos, More retoma una parte de su primera respuesta, en que Descartes advirtió que nadie ha demostrado que haya alma en los animales dado que nadie puede ingresar al corazón de éstos para comprobarlo: "hay que convenir que vos todavía no lo habéis demostrado, como vos mismo lo reconocéis, e incluso que no podéis hacerlo de ninguna manera" ${ }^{94}$. More insiste a Descartes que la mayor prueba de que los animales poseen alma es que "evitan con tanto cuidado lo que les perjudica, y que cuidan de su conservación" ${ }^{65}$, como bien se lo haría saber a través de historias asombrosas si tuviera tiempo de relatárselas. Respecto al uso de la palabra, More le pregunta a Descartes, acertadamente, si los animales podrían tener otra clase de lenguaje que dependiera de la palabra, pero que indicara la presencia de vida interior, como pasa en los niños de pocos meses:

¿Es que en los perros no nos hacen algunos signos con la cola como hacemos nosotros con la cabeza? ¿Es que con sus ladridos, no nos piden como por caridad que les pongamos su comida? Incluso empujan a veces el brazo de su amo con la pata con una contención admirable para recordarle con este signo lisonjero que les ha olvidado? ${ }^{66}$.

Además de los argumentos anteriores More aprovecha la carta para señalar otras importantes objeciones y preguntas al sistema cartesiano que agrupamos en las siguientes preguntas. En algunas de ellas es claro que More está mirando los problemas desde su propia perspectiva y no ha asumido por completo la perspectiva cartesiana, en otras, en efecto, plantea cuestiones interesantes y problemáticas para el filósofo de la Turena:

1) ¿Cómo es posible que el alma, que no tiene partes ganchudas ni ramificaciones, se puede unir tan estrechamente al cuerpo?

2) ¿No hay efectos en la naturaleza de los que no podríamos dar ninguna explicación mecánica?

3) ¿De dónde nace el sentimiento natural que tenemos de nuestra propia existencia?

4) ¿De dónde viene el dominio que nuestra alma tiene sobre los espíritus animales? ¿Cómo hace para que circulen por todas las partes del cuerpo?

5) ¿Cómo es que una sustancia incorpórea puede imprimir en cuerpo todas las propiedades del cuerpo como son el movimiento, la figura, la situación de las partes...?

64 Idem., p. 109. (More a Descartes, 5 de marzo de 1649)

65 Ibidem.

66 Correspondencia Descartes-More, p. 133. (Descartes a More, 15 de abril de 1649). 
El 15 de abril de 1649 Descartes responde a More muy rápidamente, indicándole que muchas de las respuestas a sus preguntas quedarán claras en el Tratado de las pasiones, donde "se verá claramente cómo todos los movimientos de nuestros miembros que acompañan a nuestras pasiones o afecciones son producidos, en mi opinión no por nuestra alma, sino sólo por el mecanismo del cuerpo" ${ }^{\prime 67}$. Así los movimientos que hacen los perros con la cola, explica Descartes, "no son más que movimientos que acompañan a los afectos, y creo que es necesario distinguirlos cuidadosamente de la palabra, que es la única que constituye un signo cierto del pensamiento que se oculta en el cuerpo"68.

Respecto a la comparación de More entre los niños de pocos meses y los animales, Descartes opina que hay una gran diferencia entre éstos, porque sabemos que los niños son de la misma naturaleza que los adultos, y puesto que "los animales no alcanzan jamás una edad en la que podamos darnos cuenta de que tienen el más mínimo signo de pensamiento" $"$.

La tercera carta de More a Descartes, fechada el 23 de julio de 1649, ya no toca el problema que hemos seguido y se limita a tratar otros problemas de la física, aunque le explica a Descartes su doctrina vitalista, según la cual en los cuerpos existe una "fuerza" que los hace resistirse al movimiento y que hace que las partes de los cuerpos estén unidas entre sí $^{70}$. Según Descartes, él cree que la naturaleza está conformada por espíritus extensos que penetran la materia sin llenarla y que pueden moverla (salvando el dualismo, sin abandonarlo pero evitando las dificultades de la interacción) "y todo ello sin necesidad de máquinas, de conexiones ni ganchos"71.

En agosto de 1649, en Endegeest, el francés parece haberse desesperado con la propuesta de More: una modalidad de extensión incorpórea, un espíritu de la naturaleza que daba cierta racionalidad a los seres como a los animales, era totalmente incompatible con su propuesta, donde la extensión era un atributo de la materia, $y$, por tanto, jamás podría atribuirse a una sustancia incorpórea. La pérdida de paciencia de Descartes queda expresada en el párrafo final de la misiva incompleta:

Respecto a lo que añadís acerca de que el cuerpo os parece dotado de vida, pero de una vida estúpida y llena de embriaguez, etc., todo esto me parecen muy bellas palabras; pero dejadme de una vez por todas que diga, con esta libertad con la que me habéis permitido dirigirme a vos, que nada nos aleja más del camino de la verdad que establecer ciertas cosas como verdaderas de las que no nos persuade ninguna razón positiva, sino sólo nuestra voluntad, es decir, cuando hemos inventado o imaginado algo, y vemos que nuestras ficciones nos agradan, como hacéis vos respecto a esos ángeles corpóreos, esa

67 Correspondencia Descartes-More, p. 133. (Descartes a More, 15 de abril de 1649).

68 Idem., p. 135. (Descartes a More, 15 de abril de 1649)

69 Ibidem.

70 Correspondencia Descartes-More, p. 135. (More a Descartes, 23 de julio de 1649).

71 Idem. 
sombra de la esencia divina, y otras cosas parecidas que nadie debe admitir, porque este es el mejor medio para cerrarnos todo camino a la verdad ${ }^{72}$.

\section{Conclusiones}

En las páginas anteriores hemos realizado un breve recuento de cómo se habría construido la concepción y distinción hombre-animal en la Modernidad filosófica. Nos ha interesado ahondar primeramente en el paradigma cartesiano, pues éste habría dejado importantes argumentos y supuestos para la filosofía posterior, algunos de ellos, bajo otras formulaciones, todavía podemos encontrarlos en la filosofía contemporánea. Algunas de las conclusiones cartesianas respecto al cuerpo vivo son las siguientes:

1) Que los animales no poseen alma, y por lo tanto no poseen ni razón, ni conciencia, ni lenguaje.

2) Que la explicación del movimiento animal se puede circunscribir al movimiento mecánico de los cuerpos, sin intervención del alma.

3) Que los animales no tienen sensación más allá de responder mecánicamente a los estímulos.

4) Que los animales son de muchos tipos y si se aceptara que unos tiene alma tendríamos que aceptar que todos -hasta los más imperfectos- la tienen y serían, por tanto, inmortales, lo que para Descartes es absurdo.

Respecto a la polémica de Descartes con More a través de su correspondencia, nos ha parecido interesante mostrar que ya en el siglo XVII se formulaban diversas objeciones al alejamiento del hombre de los animales y a la postura que reducía a los segundos a ser meros mecanismos. Dejar a los animales en el ámbito de la mera materialidad preocupaba a More especialmente en el ámbito de la moral, pues no queda claro a qué nos compromete su naturaleza, sin embargo, por otra parte, la propuesta cartesiana tiene la ventaja de hacer los cuerpos animales inteligibles para la ciencia. El problema principal de la polémica, según hemos visto, son las diferencias abismales entre ambos autores respecto de la concepción de la corporalidad y la estructura metafísica en que ésta se inserta. Mientras que para Descartes el cuerpo depende únicamente de la extensión, para More los cuerpo están atravesados por fuerzas vitales.

Para Descartes la diferencia radical entre los animales y los hombres es que los primeros son sólo una extensión del mundo natural, mientras que los segundos poseen alma y una vida interior comprobable a través del lenguaje. More intentará refutar los argumentos

72 Correspondencia Descartes-More, p. 173 (Descartes a More, Agosto de 1649). 
mencionando casos y experiencias particulares, lo que no es suficiente para Descartes, en tanto que no modifican los axiomas de su sistema.

La cuestión de la sensación y de la conciencia es otro tema importante que discuten estos filósofos del siglo XVII, y que hoy en día todavía se sigue discutiendo, sobre todo respecto del nivel de cercanía y compromisos a los que la sensación animal nos compromete en temas ético-jurídicos. A diferencia de Descartes y More, hoy contamos con una serie de importantes experimentos y análisis fisiológicos, pero la pregunta aún sigue abierta, aunque la balanza se haya inclinado cada vez más por los mamíferos superiores. La cuestión estriba ahora, también recordando algunos elementos de la polémica Descartes-More, en si esos elementos son determinantes para comprender la constitución animal y su relación con el hombre, o si sólo estamos repitiendo una perspectiva de valoración de la vida animal que sigue encerrada en los supuestos modernos. Pero ¿serán esos los únicos criterios válidos en esta polémica?*

\section{Bibliografía}

Aucante, Vincent, La Philosophie Médicale de Descartes, Presses Universitaires de France, Paris, 2006.

Battán, Ariela, "De Relojes, Fuentes y Molinos. La representación del cuerpo en René Descartes" en: Benítez, Laura-Robles, José Antonio (Coord.), Mecanicismo y Modernidad, Universidad del Claustro de Sor Juana, México, 2008.

Benítez Grobet, Laura, Descartes y el conocimiento del mundo natural, Porrúa, México, 2004.

Cottingham, John, Descartes, trad. de Laura Benítez (coord.), Facultad de Filosofía y Letras, Universidad Nacional Autónoma de México, México, 1995.

Descartes, René, Opera, 11 Vols., Adam, Ch. - Tannery, P. (Eds.), Vrin, Paris, 1993, (1903, $1^{\mathrm{a}}$ ed.).

nos Aires, 1975.

, Meditaciones Metafísicas, trad. de Juan Gil Fernández, Aguilar, Bue-

--------------, Los Principios de la Filosofía, trad. de Guillermo Quintás, Alianza,

Madrid, 1995.

*Artículo recibido: 25 de noviembre de 2014. Aceptado: 15 de diciembre de 2014.

Intus-Legere Filosofía / Año 2014, Vol. 8, No 2 
Leticia Flores Farfán / Rogelio Laguna

Reglas para la Dirección del Entendimiento, trad. de Aníbal Carlos Leal, Juárez Editor, Buenos Aires, 1969.

, Las Pasiones del Alma, trad. de Julián Pacho, Biblioteca Nueva, Madrid, 2005.

, El Mundo o Tratado de la Luz, trad. de Laura Benítez, Instituto de Investigaciones Filosóficas, Universidad Nacional Autónoma de México, México, 1986.

---------------------, Tratado del Hombre, trad. de Guillermo Quintás, Editora Nacional, Madrid, 1980.

-, Discurso del Método, trad. de Guillermo Quintás, Alianza, Madrid, 1999.

Garin, Eugenio, Descartes, trad. de José Martínez G., Crítica-Grijalbo, Barcelona, 1989.

Gorham, Geoffrey, "Mind-Body Dualism and the Harvey-Descartes Controversy", Journal of the History of Ideas, University of Pennsylvania, Vol. 55, No. 2, Abril de 1994, pp. 211-234.

GonZÁLEz, Recio, José Luis, "La paradoja Harvey-Descartes y el proyecto de una biología teórica", La filosofía de los científicos, 1995, pp. 61-82.

More, Henry-Descartes, René, Correspondencia, GonzÁlez Recio, José Luis (Ed.), ed. trilingüe, Antígona, Madrid, 2011.

Monroy, Zuraya, "Pensamiento animal” en: Benítez, Laura- Robles, José A. (Comps.), Homenaje a Margaret Wilson, Universidad Nacional Autónoma de México, Instituto de investigaciones Filosóficas, México, 2002.

Regan, Tom, The Case for Animal Rights, University of California Press, Berkeley, 1983.

Rodis-Lewis, Geneviève, L'Antropologie Cártesienne, Presses Universitaires de France, Paris, 1990.

Singer, Peter, "Los animales y el valor de la vida", trad. de Alejandro Herrera, en: KwiAtKwoska, Teresa- Issa, Jorge (Comps.), Los caminos de la ética ambiental, Universidad Autónoma Metropolitana, Plaza y Valdés, 1998.

Velázquez Zaragoza, Alejandra, "Las ideas cartesianas acerca de la sensación en los animales”, en: Cuaderno de Historia de la Filosofía, Proyecto Filosofía Moral y Filosofía Natural, IIF-UNAM, México, UBA, Buenos Aires, Primavera 2001, No. 4, pp. 15-21. 